\title{
Detection of high-energy heavy ions using piezoelectric lead zirconate titanate
}

\section{AUTHOR(S):}

Takechi, Seiji; Morinaga, Shin-ya; Kurozumi, Atsuma; Miyachi, Takashi; Fujii, Masayuki; Hasebe, Nobuyuki; Shibata, Hiromi; Murakami, Takeshi; Uchihori, Yukio; Okada, Nagaya

\section{CITATION:}

Takechi, Seiji ...[et al]. Detection of high-energy heavy ions using piezoelectric lead zirconate titanate. JOURNAL OF APPLIED PHYSICS 2009, 105(8): 084903.

\section{ISSUE DATE:}

2009-04

URL:

http://hdl.handle.net/2433/109897

\section{RIGHT:}

Copyright 2009 American Institute of Physics. This article may be downloaded for personal use only. Any other use requires prior permission of the author and the American Institute of Physics. The following article appeared in JOURNAL OF APPLIED PHYSICS 105, 084903 (2009) and may be found at http://link.aip.org/link/JAPIAU/v105/i8/p084903/s1 


\title{
Detection of high-energy heavy ions using piezoelectric lead zirconate titanate
}

\author{
Seiji Takechi, ${ }^{1, a)}$ Shin-ya Morinaga, ${ }^{1}$ Atsuma Kurozumi, ${ }^{1}$ Takashi Miyachi, ${ }^{2}$ Masayuki Fujii, ${ }^{2}$ \\ Nobuyuki Hasebe, ${ }^{2}$ Hiromi Shibata, ${ }^{3}$ Takeshi Murakami, ${ }^{4}$ Yukio Uchihori, ${ }^{4}$ and \\ Nagaya Okada ${ }^{5}$ \\ ${ }_{1}^{1}$ Graduate School of Engineering, Osaka City University, Osaka 558-8585, Japan \\ ${ }^{2}$ Advanced Research Institute for Science and Engineering, Waseda University, Tokyo 169-8555, Japan \\ ${ }^{3}$ Graduate School of Engineering, Kyoto University, Kyoto 606-8501, Japan \\ ${ }^{4}$ National Institute of Radiological Sciences, Chiba 263-8555, Japan \\ ${ }^{5}$ Honda Electronics Co., Ltd., Aichi 441-3193, Japan
}

(Received 2 December 2008; accepted 21 February 2009; published online 20 April 2009)

\begin{abstract}
The characteristics of a radiation detector fabricated with stacks of piezoelectric lead zirconate titanate (PZT) elements were studied by irradiating it with a $400 \mathrm{MeV} / n$ xenon (Xe) beam for various beam pulse durations. This detector is referred to as the multilayered detector (MD). To understand the production mechanism behind the output voltage obtained from the MD, measurement of the spatial distribution of the output signals generated in the MD was attempted. It was found that the amplitude observed was dependent on the number of Xe ions per unit time and the amount of ionization loss energy of Xe ions in PZT. () 2009 American Institute of Physics. [DOI: $10.1063 / 1.3106634]$
\end{abstract}

\section{INTRODUCTION}

The detection of high-energy particles via the measurement of an acoustic wave that is generated in a liquid target has been studied and the feasibility of using the acoustic method was suggested. ${ }^{1-3}$ The acoustic wave was measured by the hydrophones soaked in liquid medium. Lead zirconate titanate (PZT) may be useful as the hydrophone. Because PZT is a piezoelectric ceramic, it can be easily formed into arbitrary shapes and can be operated without a power supply because of its piezoelectricity, which means that the strain brought by an external force produces an electric charge on the surface of the material. In addition, PZT is resistant to high temperature. On the basis of the above features, we are now investigating the capability of the PZT to measure radiation. ${ }^{4-11}$

The characteristics of some detectors fabricated using PZT have been investigated with a $400 \mathrm{MeV} / n$ xenon (Xe) beam. The beam was supplied by the Heavy Ion Medical Accelerator in Chiba (HIMAC) at the National Institute of Radiological Sciences. ${ }^{12}$ These experiments have been performed with the following two methods. One is an indirect method. When the high-energy heavy ions enter a liquid material, they will lose their kinetic energy mainly due to ionization of the material. Then, the energy will be converted into heat, which generates a pressure wave via volume expansion. Consequently, the pressure wave will propagate in the material. The pressure wave was measured using the PZT detector set in the liquid material, such as water, chloroform, and ethanol, irradiated by the beam. ${ }^{5,8,10}$ The other is a direct method. When the PZT detector was directly irradiated by the beam, the electric signal generated on the PZT was monitored. ${ }^{4,6,7,9,11}$

${ }^{a)}$ Electronic mail: takechi@elec.eng.osaka-cu.ac.jp.
In our previous studies concerning the direct method, the characteristics of two types of PZT detector were studied using the $\mathrm{Xe}$ beam while varying the beam pulse duration. $^{7,9,11}$ One detector consisted of a single PZT disk with two silver $(\mathrm{Ag})$ electrodes that were coated onto the front and back surfaces of the disk. We referred to this detector as the single-layered detector (SD). The other detector was fabricated by stacking some thin PZT disks having the same diameter as the SD. A 2- $\mu$ m-thick metal sheet made of $\mathrm{Ag}-\mathrm{Pd}$ was sandwiched between each pair of disks. These disks were sintered to be the same thickness as the SD and the front and back surfaces of the assembled stack were covered with the Ag electrodes. We referred to this detector as the multilayered detector (MD). The output voltage between the two electrodes was monitored. The amplitudes of the signals obtained from the two detectors were almost independent of the beam pulse duration. This was clearly different from the result obtained for the indirect method. ${ }^{8}$ It was also found that the amplitude obtained from the MD was larger than that from the SD under the experimental conditions. The induced voltage may be considered to be due to ionization, thermal, elastic, and piezoelectric processes in the solid material. However, we still cannot explain in detail the production mechanism of the output signal from the PZT by direct interaction with the high-energy Xe beam.

In this study, when the MD was bombarded with the Xe beam, some output signals appeared on the thin PZT elements, of which the MD was built. We attempted to measure these signals while changing the beam pulse duration to a shorter range than that used so far.

\section{EXPERIMENT}

Figure 1 shows a schematic of the MD used in this experiment. The MD was fabricated using a square PZT element with a side length of $35 \mathrm{~mm}$ and a thickness of $1 \mathrm{~mm}$. 


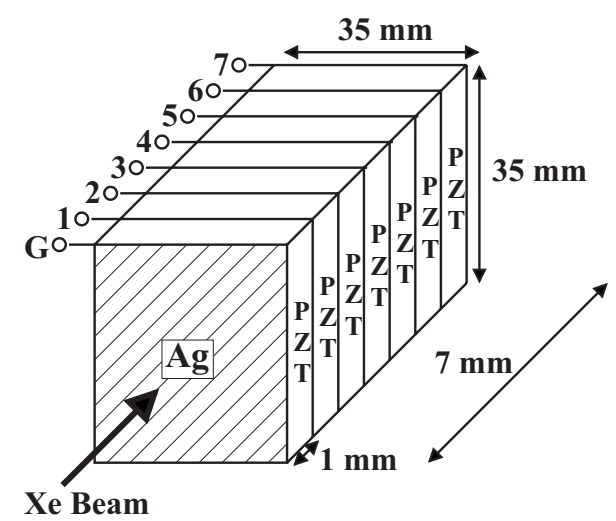

FIG. 1. Shape and dimensions of PZT MD.

The seven elements were sintered with the Ag electrode a few $\mu \mathrm{m}$ thick that was deposited on the surfaces and the total thickness of the MD was $7 \mathrm{~mm}$. The electrode on the front surface of the MD was grounded and is denoted as G in Fig. 1. The other electrodes are indicated as 1-7 from the side nearest to G, as shown in Fig. 1.

Figure 2(a) shows the experimental configuration. A

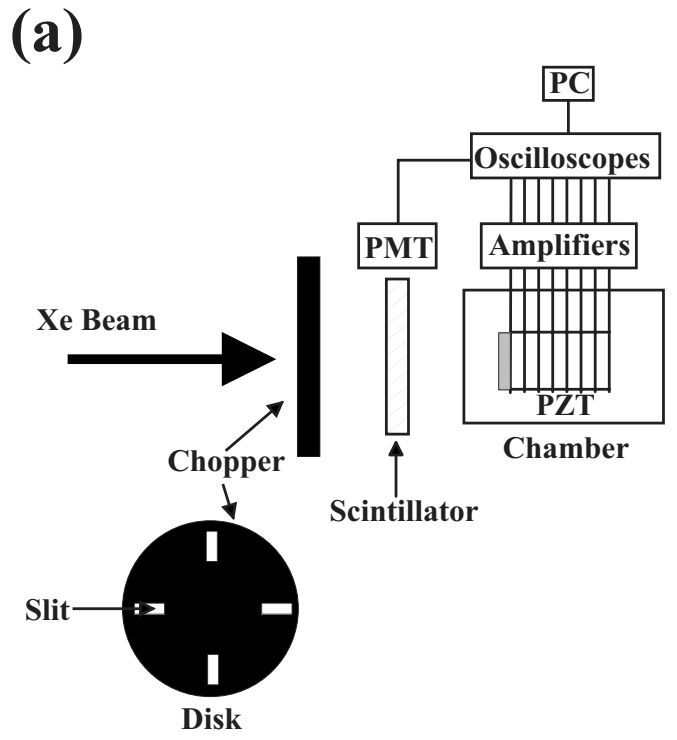

(b)

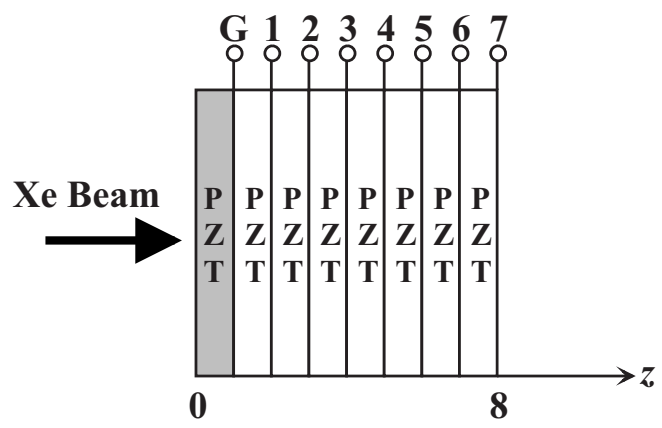

FIG. 2. (a) The experimental configuration, where the photomultiplier is indicated as PMT and the personal computer as PC. (b) Definition of $z$ position in this experiment.
$400 \mathrm{MeV} / n$ Xe beam was supplied by HIMAC. The beam was originally extracted for $0.3 \mathrm{~s}$ within a period of $3.3 \mathrm{~s}$. The Xe beam flux was fixed at $\sim 2 \times 10^{7}$ pps. In addition, a chopper was also used to create a short-pulse beam. The chopper was a rotating stainless-steel disk with a diameter of $30 \mathrm{~cm}$ and a thickness of $10 \mathrm{~mm}$ with four equiangular slits with a length of $10 \mathrm{~mm}$ and a width of $1 \mathrm{~mm}$. The radial position of the slits in the chopper disk was $\sim 14 \mathrm{~cm}$. The chopper was installed $\sim 40 \mathrm{~cm}$ downstream of the exit of the beam duct. By adjusting the rotation speed, the number of xenon ions could be varied in accordance with the change in pulse duration. The beam passing through the slit was monitored using a 1-mm-thick plastic scintillator with a photomultiplier (PMT), placed $\sim 69 \mathrm{~cm}$ downstream of the chopper. The signal detected by the PMT was used not only as a time reference but also as a standard of the beam intensity in this experiment. A frame made of epoxy resin supported the MD. The frame was suspended from four springs to prevent noise arising from mechanical disturbances. Moreover, to eliminate noise originating from the variation in atmospheric pressure, the MD was set in a closed chamber. The chamber, which was made of transparent acrylic resin to enable visual confirmation of the alignment, was placed $\sim 13 \mathrm{~cm}$ downstream of the scintillator. When the Xe beam was irradiated onto the MD, the seven output voltages from between $\mathrm{G}$ and 1 to between $\mathrm{G}$ and 7 were monitored. Each output signal was processed using the respective amplifier and then transferred to two digital oscilloscopes. Note that the output between $\mathrm{G}$ and 3 could not be utilized because the amplifier was out of order. Digitally processed data were stored in a personal computer.

An additional PZT element of $1 \mathrm{~mm}$ thick was placed in front of the MD to confine the Xe within the MD; it is represented as the shaded element in Fig. 2(b). The range of $400 \mathrm{MeV} / n \mathrm{Xe}$ in PZT was estimated to be less than $\sim 8 \mathrm{~mm}$ using the Bethe-Bloch formula. Figure 2(b) shows the definition of spatial coordinate $z$ in this experiment. The Xe beam was incident on the center of the first PZT element, where the beam had a diameter of $\sim 4 \mathrm{~mm}$.

\section{RESULTS AND DISCUSSION}

Figure 3 shows typical output waveforms when the rotation speed of the chopper was $60 \mathrm{rps}$, where Fig. 3(a) shows the scintillator signal obtained from the PMT and Fig. 3(b) shows the signal obtained from between $G$ and 7. Note that these waveforms were reconstructed by inverse Fourier transformation using the frequency components below 25 $\mathrm{kHz}$ to eliminate the noise signal. Here, the time at which the shutter for controlling the Xe beam passage was opened is defined as $t_{0}$ and the time at which the shutter was closed is defined as $t_{1}$, that is, the time during which the beam was irradiated onto the MD is defined as $T_{L}=t_{1}-t_{0}$. These are indicated in Fig. 3(a). Note that the $T_{L}$ may not be just duration of the beam pulse. However it was confirmed that the time during which the electric signal appeared on the PMT was linearly related to the pulse duration in the previous 

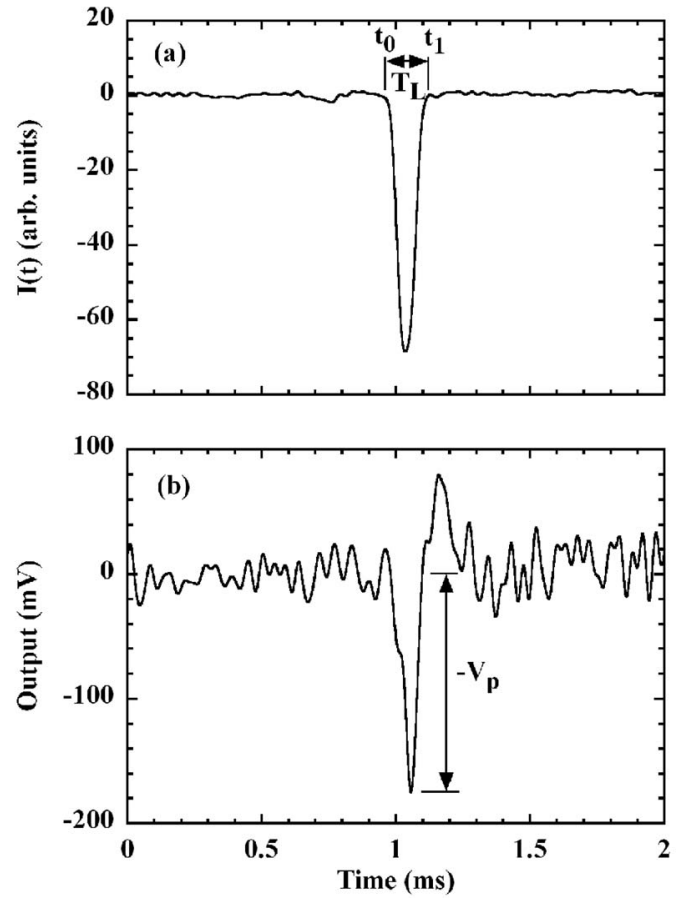

FIG. 3. Typical output waveforms obtained from (a) PMT and (b) MD, which are reconstructed by inverse Fourier transformation using frequency components below $25 \mathrm{kHz}$.

work. The amplitude of the signal obtained between two terminals in the MD is defined as $V_{p}$, which is indicated in Fig. 3(b).

Figure 4 shows the parameters estimated from the PMT signal when the rotation speed of the chopper was changed from 30 to $80 \mathrm{rps}$, where Fig. 4(a) shows the value of $T_{L}$ and Fig. 4(b) shows an integral value of the light emission over $T_{L}$, that is, $I_{L}=\int_{t_{0}}^{t_{1}}|I(t)| d t$. Note that the closed circle indicates
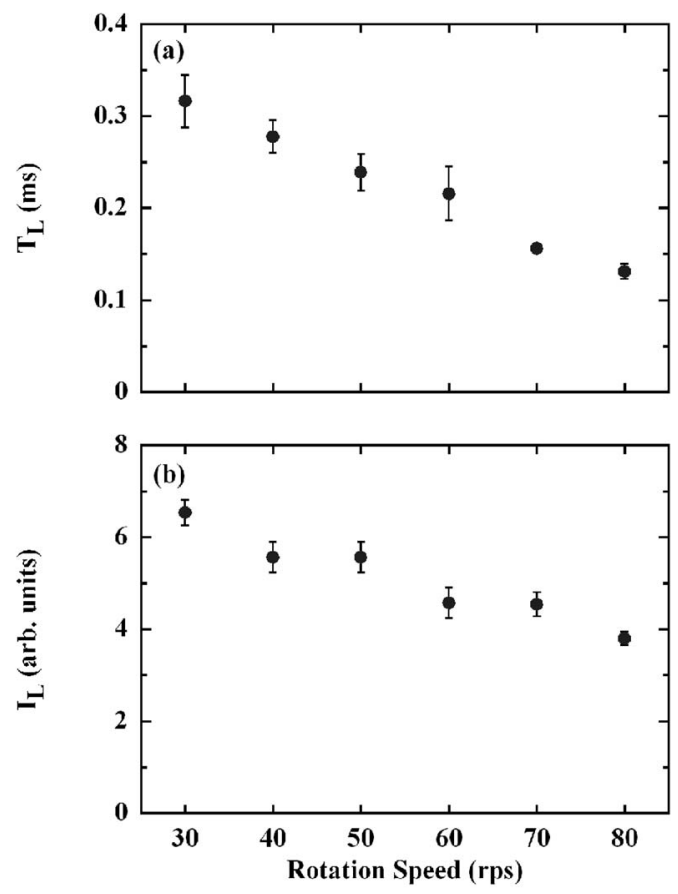

FIG. 4. Dependence of (a) $T_{L}$ and (b) $I_{L}$ on rotation speed of chopper.
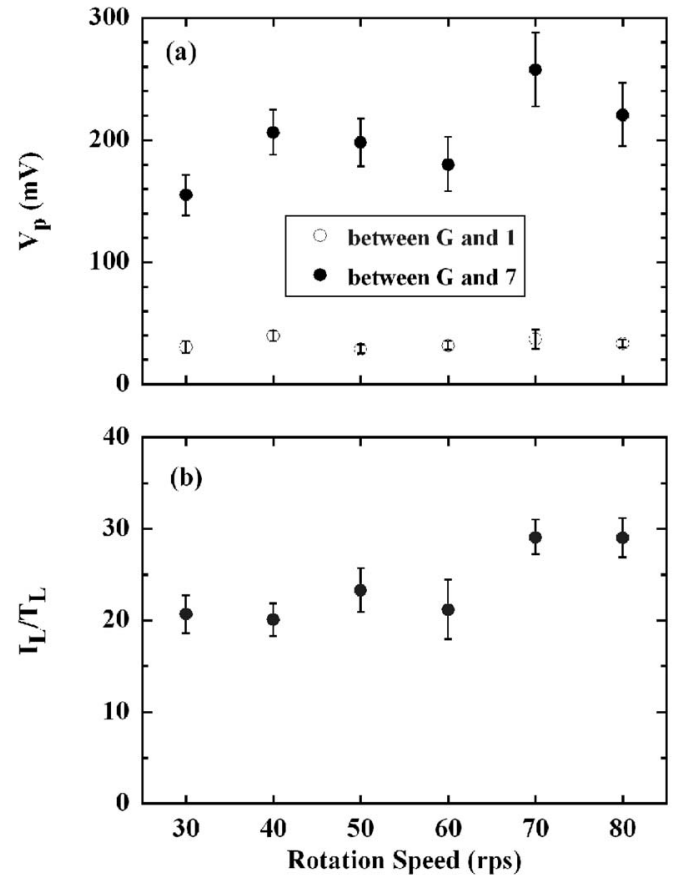

FIG. 5. Dependence of (a) $V_{p}$ obtained between $\mathrm{G}$ and 1 and between $\mathrm{G}$ and 7 , and (b) $I_{L} / T_{L}$ on rotation speed of chopper.

a value averaged over $\sim 30$ events and the vertical error bar indicates the standard deviation. In this experiment, we took one event within $0.3 \mathrm{~s}$. It can be seen from Figs. 4(a) and 4(b) that $T_{L}$ was shorter and $I_{L}$ was smaller with increasing rotation speed of the chopper. Figure 4(b) indicates that the Xe beam intensity, that is, the number of the Xe ions, became lower as the rotation speed increased.

Figure 5(a) shows the value of $V_{p}$ obtained from the MD when the rotation speed of the chopper was changed from 30 to $80 \mathrm{rps}$. The open circle is for the signal from between $\mathrm{G}$ and 1 and the closed circle is for that from between $\mathrm{G}$ and 7 . These values were the averages of $\sim 30$ events and the vertical error bar indicates the standard deviation. The $V_{p}$ obtained for the signal from between $\mathrm{G}$ and 1 was almost independent of the rotation speed. On the other hand, the $V_{p}$ obtained for the signal from between $\mathrm{G}$ and 7 seemed to become larger as the rotation speed increased. Note that the previous results for the direct method ${ }^{7,9,11}$ were obtained with the rotation speed up to $\sim 55 \mathrm{rps}$ and the results did not show explicit dependence within the limits of the speed. This seems to be also true of the one seen from Fig. 5(a). When the rotation speed was extended to a faster range than that used so far, it was first confirmed that, for the direct method, the amplitude observed was dependent on the rotation speed. Figure 5(b) shows the beam intensity per unit time, that is, $I_{L} / T_{L}$, with the change in rotation speed from 30 to $80 \mathrm{rps}$. The number of $\mathrm{Xe}$ ions per unit time became larger with increasing rotation speed. This may be reflected in the value of $V_{p}$ obtained for the signal from between $\mathrm{G}$ and 7 . The pressure wave generated in a liquid target is considered to be proportional to the time differential of the amount of energy deposited into the target. ${ }^{1}$

Figure 6 shows the spatial distribution of $V_{p}$ obtained from the MD, except that for the signal from between $G$ and 


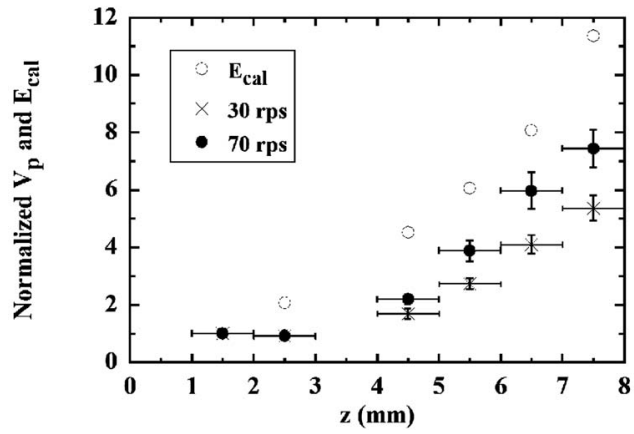

FIG. 6. Spatial distributions of $V_{p}$ obtained from MD when the rotation speeds were 30 and $70 \mathrm{rps}$, respectively, and $E_{\text {cal }}$ is calculated using the Bethe-Bloch formula. The values of $V_{p}$ are normalized to that obtained between $\mathrm{G}$ and 1 and those of $E_{\text {cal }}$ are also normalized to that calculated for Xe traveling from $z=1$ to $2 \mathrm{~mm}$ in PZT.

3 , when the rotation speed was 30 and $70 \mathrm{rps}$, respectively. Note that the value of $V_{p}$ was normalized to that between $\mathrm{G}$ and 1 . The horizontal error bar indicates a variation of $1 \mathrm{~mm}$, corresponding to the thickness of the PZT element. The open circle represents the calculated value using the Bethe-Bloch formula as $E_{\text {cal }}$, which corresponds to the energy that a $400 \mathrm{MeV} / n$ Xe ion loses in PZT owing to ionization as the $\mathrm{Xe}$ ion travels from $z=1 \mathrm{~mm}$ to the surface of each element. Note that the value of $E_{\text {cal }}$ was also normalized to that calculated at distances from $z=1 \mathrm{~mm}$ to $z=2 \mathrm{~mm}$, which is equal to $\sim 0.7 \mathrm{~nJ}$. Although there was difference in $I_{L} / T_{L}$ as seen from Fig. 5(b), the difference between the two $V_{p}$ values began to appear when the Xe ion went into $z=5 \mathrm{~mm}$. This may be attributed to resolving power of the PZT detector used. It was found that $V_{p}$ became larger with increasing $E_{\text {cal }}$, on the other hand, the space differential of the $V_{p}$ seemed to be different from that of the $E_{\mathrm{cal}}$. Note that the experimental value was measured for the ion flux and the calculation was performed for the single ion. To clarify more quantitatively how the Xe energy lost by interaction with PZT is transformed to the output signal, the measurement for the single ion using a detector with higher sensitivity is needed.

\section{CONCLUSION}

To understand the production mechanism of the output voltage appearing on the PZT MD, we examined the output signal generated on each element that constitutes the MD when a $400 \mathrm{MeV} / n$ Xe beam was directly irradiated onto the MD, while changing the rotation speed of the chopper up to 80 rps. It was first confirmed that the amplitude of the output signal obtained from the detector was dependent on the beam pulse duration. This result may be partly due to the number of Xe ions per unit time. Compared with the result calculated using the Bethe-Bloch formula, the above dependence appeared within a range at which the ionization loss energy of the Xe ion in PZT was estimated to be greater than $\sim 3 \mathrm{~nJ}$. It was also found that the total energy of the Xe beam was not always reflected on the output voltage. For simplicity, a better PZT detector that enables the measurement of a single Xe ion needs to be developed. It use will lead to a more quantitative understanding of the production mechanism of the output voltage appearing on PZT.

\section{ACKNOWLEDGMENTS}

This study was carried out as part of the Research Project with Heavy Ions at NIRS-HIMAC. It was partly supported by Grants-in-Aid from JSPS.

${ }^{1}$ L. Sulak, T. Armstrong, H. Baranger, M. Bergman, T. Bowen, A. E. Pier, P. A. Polakos, H. Bradner, A. Parvulescu, W. V. Jones, and J. Learned, Nucl. Instrum. Methods 161, 203 (1979).

${ }^{2}$ G. A. Askariyan, B. A. Dolgoshein, A. N. Kalinovsky, and N. V. Mokhov, Nucl. Instrum. Methods 164, 267 (1979).

${ }^{3}$ L. G. Dedenko, I. V. Denisov, A. A. Mironovich, A. L. Provorov, and I. M. Zheleznykh, PACON 99 Proceedings of the Symposium on Humanity and the World Ocean, Moscow, Russia, 1999 (unpublished), p. 535.

${ }^{4}$ T. Miyachi, N. Hasebe, H. Ito, T. Masumura, H. Okada, O. Okudaira, N. Yamashita, H. Yoshioka, M. Higuchi, T. Matsuyama, T. Murakami, Y. Uchihori, M. Kase, R. Kikuchi, M. Sato, and T. Tou, Jpn. J. Appl. Phys., Part 1 42, 1456 (2003).

${ }^{5}$ T. Miyachi, Y. Nakamura, G. Kuraza, M. Fujii, A. Nagashima, N. Hasebe, M. N. Kobayashi, S. Kobayashi, M. Miyajima, O. Okudaira, N. Yamashita, H. Shibata, T. Murakami, Y. Uchihori, N. Okada, and T. Tou, Nucl. Instrum. Methods Phys. Res. A 560, 606 (2006).

${ }^{6}$ T. Miyachi, Y. Nakamura, G. Kuraza, M. Fujii, A. Nagashima, N. Hasebe, M. N. Kobayashi, S. Kobayashi, M. Miyajima, K. Mori, O. Okudaira, N. Yamashita, H. Shibata, T. Murakami, Y. Uchihori, and N. Okada, Nucl. Instrum. Methods Phys. Res. A 568, 760 (2006).

${ }^{7}$ S. Takechi, T. Onishi, S. Minami, T. Miyachi, M. Fujii, N. Hasebe, K. Mori, H. Shibata, T. Murakami, Y. Uchihori, and N. Okada, Jpn. J. Appl. Phys., Part 1 46, 1704 (2007).

${ }^{8}$ S. Takechi, T. Onishi, S. Minami, T. Miyachi, M. Fujii, N. Hasebe, K. Mori, H. Shibata, T. Murakami, Y. Uchihori, and N. Okada, Nucl. Instrum. Methods Phys. Res. A 577, 729 (2007).

${ }^{9}$ S. Takechi, T. Onishi, S. Minami, T. Miyachi, M. Fujii, N. Hasebe, K. Mori, H. Shibata, T. Murakami, Y. Uchihori, and N. Okada, Nucl. Instrum. Methods Phys. Res. A 577, 741 (2007).

${ }^{10}$ S. Takechi, T. Miyachi, M. Fujii, N. Hasebe, K. Mori, H. Shibata, T. Murakami, Y. Uchihori, and N. Okada, Nucl. Instrum. Methods Phys. Res. A 586, 309 (2008).

${ }^{11}$ S. Takechi, T. Miyachi, M. Fujii, N. Hasebe, K. Mori, H. Shibata, T. Murakami, Y. Uchihori, and N. Okada, Sens. Actuators, A 147, 365 (2008).

${ }^{12}$ Y. Hirao, H. Ogawa, S. Yamada, Y. Goto, T. Yamada, K. Sato, A. Itano, M. Kanazawa, K. Noda, K. Kawachi, M. Endo, T. Kanai, T. Kohno, M. Sudou, S. Minohara, A. Kitagawa, F. Soga, E. Takada, S. Watanabe, K. Endo, M. Kumada, and S. Matsumoto, Nucl. Phys. A. 538, 541 (1992). 